Dynamic Systems and Applications 16 (2007) 337-344

\title{
INTERVAL OSCILLATION CRITERIA FOR SECOND ORDER NONLINEAR DIFFERENTIAL EQUATIONS WITH DAMPING
}

\author{
YURI V. ROGOVCHENKO AND FATOŞ TUNCAY \\ Department of Mathematics, Eastern Mediterranean University, Famagusta, \\ TRNC, Mersin 10, Turkey and Department of Chemistry and Biomedical Sciences, \\ University of Kalmar, SE-39182 Kalmar, Sweden \\ Department of Mathematics, Eastern Mediterranean University, Famagusta, \\ TRNC, Mersin 10, Turkey
}

\begin{abstract}
Using generalized Riccati transformations, for a second order nonlinear differential equation with a damping term we derive nontrivial extensions of oscillation criteria obtained for linear equations by Kong [J. Math. Anal. Appl. 229 (1999), 258-270]. Efficiency of new results is illustrated on the example extracted from the recent paper by Sun [J. Math. Anal. Appl. 291 (2004), 341-351].
\end{abstract}

AMS (MOS) Subject Classification. 34C10.

\section{INTRODUCTION}

Recently, problems regarding oscillation and nonoscillation for different classes of nonlinear second order differential equations with damping terms attracted attention of many researchers, see, e.g., the monographs $[1,2]$ and the papers [4]-[6], [8], [10][11], [13]-[16], [18]-[22] where further references can be found.

In this paper, we are concerned with the oscillatory nature of a second order nonlinear differential equation

$$
\left(r(t) x^{\prime}(t)\right)^{\prime}+p(t) x^{\prime}(t)+q(t) f(x(t))=0
$$

where $r \in C^{1}\left(\left[t_{0},+\infty\right),(0,+\infty)\right), p, q \in C\left(\left[t_{0},+\infty\right), \mathbb{R}\right), f \in C(\mathbb{R}, \mathbb{R})$, and $x f(x)>0$ for all $x \neq 0$. Our goal is to derive for equation (1.1) efficient and easily verifiable sufficient conditions for the oscillation which use information on the behavior of coefficients of equation on an infinite sequence of intervals rather than on the entire positive semi-axis.

First results of this type were obtained by El-Sayed [3] and later by Huang [7], Kong [9], Li and Agarwal [10], Nasr [12], Sun [16, 17], Tiryaki and Zafer [18], and Zheng [22]. These criteria, called interval oscillation theorems, are especially efficient 
in situations when, for instance,

$$
\int^{+\infty} q(s) d s=-\infty
$$

and many advanced oscillation results based on the standard integral averaging technique fail to apply. A typical example of this kind extracted from the recent paper by Sun [16, Example 2, p. 350] is considered in the final part of the paper to test the efficiency of new results.

\section{OSCILLATION CRITERIA}

Let $D=\{(t, s) \mid-\infty<s \leq t<+\infty\}$. In what follows, we say that a continuous function $H(t, s), H: D \rightarrow[0,+\infty)$, belongs to the class $\mathcal{W}$ if

(i) $H(t, t)=0$ and $H(t, s)>0$ for $-\infty<s<t<+\infty$;

(ii) $H$ has continuous partial derivatives $\partial H / \partial t$ and $\partial H / \partial s$ satisfying

$$
\frac{\partial H}{\partial t}=h_{1}(t, s) \sqrt{H(t, s)} \quad \text { and } \quad \frac{\partial H}{\partial s}=-h_{2}(t, s) \sqrt{H(t, s)},
$$

where $h_{1}, h_{2} \in L_{\mathrm{loc}}(D, \mathbb{R})$.

The following technical result is a particular case of [18, Lemma 1.1].

Lemma 2.1. Suppose that a function $u \in C^{1}(D, \mathbb{R})$ satisfies, for all $t \in(a, b) \subset$ $\left[t_{0},+\infty\right)$, the inequality

$$
u^{\prime}(t) \leq-\alpha(t)-\beta(t) u^{2}(t)
$$

where $\alpha \in C\left(\left[t_{0},+\infty\right), \mathbb{R}\right), \beta \in C\left(\left[t_{0},+\infty\right),(0,+\infty)\right)$. Then, for any $c \in(a, b)$ and for any $H \in \mathcal{W}$,

$$
\begin{aligned}
\frac{1}{H(c, a)} \int_{a}^{c}\left[\alpha(s) H(s, a)-\frac{1}{4 \beta(s)} h_{1}^{2}(s, a)\right] d s & \\
& +\frac{1}{H(b, c)} \int_{c}^{b}\left[\alpha(s) H(b, s)-\frac{1}{4 \beta(s)} h_{2}^{2}(b, s)\right] d s \leq 0 .
\end{aligned}
$$

Theorem 2.2. Suppose that $f$ is continuously differentiable on $\mathbb{R}$ and

$$
f^{\prime}(x) \geq \mu>0,
$$

for all $x \in \mathbb{R}$. Assume also that there exists a function $g \in C^{1}\left(\left[t_{0},+\infty\right), \mathbb{R}\right)$ such that, for some $H \in \mathcal{W}$ and $c \in(a, b)$,

$$
\begin{aligned}
& \frac{1}{H(c, a)} \int_{a}^{c}\left[H(s, a) \varphi(s)-\frac{1}{4 \mu} v(s) r(s) h_{1}^{2}(s, a)\right] d s \\
& >\frac{1}{H(b, c)} \int_{c}^{b}\left[\frac{1}{4 \mu} v(s) r(s) h_{2}^{2}(b, s)-H(b, s) \varphi(s)\right] d s
\end{aligned}
$$

where

$$
v(t)=\exp \left[\int^{t}\left(\frac{p(s)}{r(s)}-2 \mu g(s)\right) d s\right]
$$


and

$$
\varphi(t)=v(t)\left[q(t)+\mu r(t) g^{2}(t)-p(t) g(t)-(r(t) g(t))^{\prime}\right] .
$$

Then, every solution of equation (1.1) has at least one zero in $(a, b)$.

Proof. For the sake of contradiction, assume that there exists a solution $x(t)$ of equation (1.1) that has no zeros in $(a, b)$. Without loss of generality, we can suppose that $x(t)>0$ for $t \in(a, b)$. Let $g \in C^{1}\left(\left[t_{0},+\infty\right), \mathbb{R}\right)$ and $v$ be defined by (2.3). Introduce a new variable $u$ by

$$
u(t)=v(t) r(t)\left[\frac{x^{\prime}(t)}{f(x(t))}+g(t)\right] .
$$

Note that $u$ is well defined because with the above choice of $x(t), f(x(t))>0$ for $t \in(a, b)$. Differentiating (2.5), we obtain

$$
u^{\prime}(t)=\frac{v^{\prime}(t)}{v(t)} u(t)+v(t)\left\{\frac{\left[r(t) x^{\prime}(t)\right]^{\prime}}{f(x(t))}-\frac{r(t)\left[x^{\prime}(t)\right]^{2} f^{\prime}(x(t))}{[f(x(t))]^{2}}+[r(t) g(t)]^{\prime}\right\} .
$$

Using (1.1), (2.1), (2.3), and (2.5), one has

$$
\begin{aligned}
u^{\prime}(t) \leq\left[-2 \mu g(t)+\frac{p(t)}{r(t)}\right] u(t)+ & v(t)\left\{-p(t)\left[\frac{u(t)}{v(t) r(t)}-g(t)\right]\right. \\
& \left.-q(t)-\mu r(t)\left[\frac{u(t)}{v(t) r(t)}-g(t)\right]^{2}+[r(t) g(t)]^{\prime}\right\},
\end{aligned}
$$

which, after elementary algebra, leads to

$$
u^{\prime}(t) \leq-\varphi(t)-\frac{\mu}{v(t) r(t)} u^{2}(t)
$$

where $\varphi(t)$ is defined by (2.4). Applying to (2.6) Lemma 2.1 with $\alpha(t)=\varphi(t)$ and $\beta(t)=\mu(v(t) r(t))^{-1}$, one has, for any $c \in(a, b)$ and for any $H \in \mathcal{W}$,

$$
\begin{gathered}
\frac{1}{H(c, a)} \int_{a}^{c}\left[\varphi(s) H(s, a)-\frac{v(s) r(s)}{4 \mu} h_{1}^{2}(s, a)\right] d s \\
+\frac{1}{H(b, c)} \int_{c}^{b}\left[\varphi(s) H(b, s)-\frac{v(s) r(s)}{4 \mu} h_{2}^{2}(b, s)\right] d s \leq 0,
\end{gathered}
$$

which contradicts $(2.2)$. The proof is complete.

The following result is an immediate consequence of Theorem 2.2.

Theorem 2.3. Let (2.1) hold and, for any $\tau \geq t_{0}$, there exist a function $g \in$ $C^{1}\left(\left[t_{0},+\infty\right), \mathbb{R}\right)$ and real numbers $a, b, \tau \leq a<b$ such that, for some $H \in \mathcal{W}$ and $c \in(a, b),(2.2)$ is satisfied with $v$ and $\varphi$ defined by (2.3) and (2.4) respectively. Then, equation (1.1) is oscillatory. 
Proof. Pick a sequence of real numbers, $t_{0} \leq \tau_{1} \leq \tau_{2} \leq \cdots$, satisfying $\tau_{n} \rightarrow+\infty$ as $n \rightarrow+\infty$. By the assumptions of the theorem, for any $\tau_{n}$, there exist a function $g \in C^{1}\left(\left[t_{0},+\infty\right), \mathbb{R}\right)$ and a pair of real numbers $a_{n}, b_{n}, \tau_{n} \leq a_{n}<b_{n}$ such that, for some $H \in \mathcal{W}$ and $c_{n} \in\left(a_{n}, b_{n}\right)$,

$$
\begin{aligned}
\frac{1}{H\left(c_{n}, a_{n}\right)} \int_{a_{n}}^{c_{n}}\left[H\left(s, a_{n}\right) \varphi(s)-\frac{1}{4 \mu} v(s) r(s) h_{1}^{2}\left(s, a_{n}\right)\right] d s \\
>\frac{1}{H\left(b_{n}, c_{n}\right)} \int_{a_{n}}^{c_{n}}\left[\frac{1}{4 \mu} v(s) r(s) h_{2}^{2}\left(b_{n}, s\right)-H\left(b_{n}, s\right) \varphi(s)\right] d s .
\end{aligned}
$$

Then, by virtue of Theorem 2.2, any solution $x(t)$ of equation (1.1) has at least one zero in $\left(a_{n}, b_{n}\right)$. Taking into account that $a_{n} \rightarrow+\infty$ and $b_{n} \rightarrow+\infty$ as $n \rightarrow+\infty$, we conclude that the sequence of zeros of arbitrary solution $x(t)$ of equation (1.1) diverges to $+\infty$, that is, $x(t)$ oscillates. Therefore, equation (1.1) is oscillatory.

In applications, a class of difference kernels $\mathcal{W}_{0} \subset \mathcal{W}$ plays an important role. It contains all functions $H \in \mathcal{W}$ that satisfy $H(t, s)=H(t-s)$, in which case $h_{1}(t-s)=h_{2}(t-s) \stackrel{\text { def }}{=} h(t-s)$. For the class $\mathcal{W}_{0}$, a consequence of Theorem 2.3 is the following result.

Theorem 2.4. Let (2.1) hold and, for any $\tau \geq t_{0}$, there exist a function $g \in$ $C^{1}\left(\left[t_{0},+\infty\right), \mathbb{R}\right)$ and real numbers $a, c, \tau \leq a<c$ such that, for some $H \in \mathcal{W}_{0}$,

$$
\begin{aligned}
& \int_{a}^{c} H(s-a)[\varphi(s)+\varphi(2 c-s)] d s \\
& >\frac{1}{4 \mu} \int_{a}^{c}[v(s) r(s)+v(2 c-s) r(2 c-s)] h^{2}(s-a) d s,
\end{aligned}
$$

where $v$ and $\varphi$ are as in Theorem 2.2. Then, equation (1.1) is oscillatory.

Proof. In Theorem 2.3, let $c$ be the midpoint of the interval $[a, b], c=(a+b) / 2$. Then,

$$
\begin{gathered}
\frac{1}{H(c-a)} \int_{a}^{c} H(s-a) \varphi(s) d s+\frac{1}{H(b-c)} \int_{c}^{b} H(b-s) \varphi(s) d s \\
=\frac{1}{H(c-a)} \int_{a}^{c} H(s-a)[\varphi(s)+\varphi(2 c-s)] d s \\
\frac{1}{H(c-a)} \int_{a}^{c}\left[v(s) r(s) h^{2}(s-a)\right] d s+\frac{1}{H(b-c)} \int_{c}^{b}\left[v(s) r(s) h^{2}(b-s)\right] d s \\
=\frac{1}{H(c-a)} \int_{a}^{c}[v(s) r(s)+v(2 c-s) r(2 c-s)] h^{2}(s-a) d s,
\end{gathered}
$$

and (2.8) is equivalent to (2.2). Thus, equation (1.1) is oscillatory by Theorem 2.3.

Choosing in Theorem 2.4

$$
H(t, s)=(t-s)^{n}, n>1
$$


one arrives at a Kamenev-type oscillation criterion.

Corollary 2.5. Assume that (2.1) holds and, for any $\tau \geq t_{0}$, there exist a function $g \in C^{1}\left(\left[t_{0},+\infty\right), \mathbb{R}\right)$ and real numbers $a, c, \tau \leq a<c$ such that

$$
\begin{aligned}
\int_{a}^{c}(s-a)^{n}[\varphi(s)+\varphi(2 c-s)] & d s \\
> & \frac{n^{2}}{4 \mu} \int_{a}^{c}[v(s) r(s)+v(2 c-s) r(2 c-s)](s-a)^{n-2} d s,
\end{aligned}
$$

where $v$ and $\varphi$ are as in Theorem 2.2. Then, equation (1.1) is oscillatory.

Suppose now that $f$ is continuous on $\mathbb{R}$ and satisfies, for all $x \in \mathbb{R}$,

$$
\frac{f(x)}{x} \geq \mu>0 \text {. }
$$

Consequently, $f$ may not necessarily be increasing and may not even be differentiable. In this case, one needs an additional condition,

$$
q(t) \geq 0
$$

for all $t \geq t_{0}$, and $q(t)$ should not be identical zero on $\left[t_{0},+\infty\right)$.

Theorem 2.6. Assume that (2.9), (2.10) are satisfied, and there exists a function $g \in C^{1}\left(\left[t_{0},+\infty\right), \mathbb{R}\right)$ such that, for some $H \in \mathcal{W}$ and $c \in(a, b),(2.2)$ holds, where $v$ and $\varphi$ are defined as in Theorem 2.2. Then, every solution of equation (1.1) has at least one zero in $(a, b)$.

Proof. As in Theorem 2.2, assume, without loss of generality, that there exists a solution $x(t)$ of equation (1.1) such that $x(t)>0$ for $t \in(a, b)$. Let $g \in C^{1}\left(\left[t_{0},+\infty\right), \mathbb{R}\right)$ and $v$ be defined by (2.3). Introduce a new variable $u$ by

$$
u(t)=v(t) r(t)\left[\frac{x^{\prime}(t)}{x(t)}+\mu g(t)\right]
$$

and note that $u$ is well defined because $x(t)>0$ for $t \in(a, b)$. Differentiating equation (2.11) and proceeding as in the proof of Theorem 2.2, one arrives at

$$
u^{\prime}(t) \leq-\mu \varphi(t)-\frac{1}{v(t) r(t)} u^{2}(t) .
$$

An application of Lemma 2.1 yields, for any $c \in(a, b)$ and for any $H \in \mathcal{W}$, inequality (2.7), which contradicts (2.2). Hence, any solution of equation (1.1) has at least one zero in $(a, b)$.

Theorem 2.7. Assume that (2.9), (2.10) are satisfied, and, for any $\tau \geq t_{0}$, there exist a function $g \in C^{1}\left(\left[t_{0},+\infty\right), \mathbb{R}\right)$ and real numbers $a, b, \tau \leq a<b$ such that, for some $H \in \mathcal{W}$ and $c \in(a, b)$, (2.2) holds with $v$ and $\varphi$ defined as in Theorem 2.2. Then, equation (1.1) is oscillatory. 
Proof. The proof runs as in Theorem 2.3 with obvious modifications.

Remark 2.8. It is not difficult to obtain results similar to Theorem 2.4 and Corollary 2.5, as well as other oscillation criteria with different choices of $H(t, s)$. We point out that a number of alike results that can be obtained by using generalized Riccati substitutions different from (2.5) and (2.11) are actually nothing else but variations of Theorems 2.2 and 2.6. Indeed, using instead of (2.5) the transformation

$$
u(t)=v(t) r(t)\left[\alpha \frac{x^{\prime}(t)}{f(x(t))}+\beta g(t)\right],
$$

where $\alpha>0$ and $\beta \in \mathbb{R}$, one has to pick

$$
v(t)=\exp \left[\int^{t}\left(\frac{p(s)}{r(s)}-\frac{2 \mu \beta}{\alpha} g(s)\right) d s\right]
$$

and adjust $\varphi$ accordingly. A similar reasoning applies to possible replacements for (2.11).

Example 2.9. Consider the nonlinear differential equation examined in the recent paper by Sun [16, Example 2, p. 350],

$$
x^{\prime \prime}+p(t) x^{\prime}+q(t)\left(x+x^{3}\right)=0, \quad t \geq t_{0},
$$

where

$$
\sqrt{\gamma} p(t)=q(t)= \begin{cases}\gamma(t-3 n), & 3 n \leq t \leq 3 n+1 \\ -\gamma(t-(3 n+2)), & 3 n+1<t \leq 3 n+2, \\ n(t-(3 n+3))(t-(3 n+2)), & 3 n+2<t \leq 3 n+3\end{cases}
$$

Note that in this example

$$
\int^{+\infty} p(t) d t=\int^{+\infty} q(t) d t=-\infty,
$$

and oscillation criteria obtained by using standard integral averaging technique do not apply. Sun proved that equation (2.12) is oscillatory by [16, Corollary 3.3, p. 349] for "sufficiently large" $\gamma$ satisfying

$$
\gamma>\frac{1729+7 \sqrt{3409}}{72} \approx 29.7
$$

whereas an application of Corollary 2.5 with $v(t)=1$ guarantees oscillation of equation (2.12) for $\gamma$ satisfying

$$
\gamma>\frac{3680+40 \sqrt{895}}{841} \approx 5.8
$$




\section{REFERENCES}

[1] R.P. Agarwal, S.R. Grace, and D. O'Regan, Oscillation Theory for Difference and Functional Differential Equations, Kluwer, Dordrecht, 2000.

[2] R.P. Agarwal, S.R. Grace, and D. O'Regan, Oscillation Theory for Second Order Linear, HalfLinear, Superlinear and Sublinear Dynamic Equations, Kluwer, Dordrecht, 2002.

[3] M.A. El-Sayed, An oscillation criterion for a forced second-order linear differential equation, Proc. Amer. Math. Soc. 118 (1993), 813-817.

[4] S.R. Grace, Oscillation theorems for second order nonlinear differential equations with damping, Math. Nachr. 141 (1989), 117-127.

[5] S.R. Grace, Oscillation theorems for nonlinear differential equations of second order, J. Math. Anal. Appl. 171 (1992), 220-241.

[6] S.R. Grace and B.S. Lalli, Integral averaging techniques for the oscillation of second order nonlinear differential equations, J. Math. Anal. Appl. 149 (1990), 277-311.

[7] C.C. Huang, Oscillation and nonoscillation for second order linear differential equations, $J$. Math. Anal. Appl. 210 (1997), 712-723.

[8] M. Kirane and Yu.V. Rogovchenko, Oscillation results for second order damped differential equation with nonmonotonous nonlinearity, J. Math. Anal. Appl. 250 (2000), 118-138.

[9] Q. Kong, Interval criteria for oscillation of second-order linear ordinary differential equations, J. Math. Anal. Appl. 229 (1999), 258-270.

[10] W.T. Li and R.P. Agarwal, Interval oscillation criteria for second order nonlinear differential equations with damping, Computers and Math. Appl. 40 (2000), 217-230.

[11] O.G. Mustafa, S.P. Rogovchenko, and Yu.V. Rogovchenko, On oscillation of nonlinear secondorder differential equations with damping term, J. Math. Anal. Appl. 298 (2004), 604-620.

[12] A.H. Nasr, Sufficient conditions for the oscillation of forced super-linear second-order differential equations with oscillatory potential, Proc. Amer. Math. Soc. 126 (1998), 123-125.

[13] S.P. Rogovchenko and Yu.V. Rogovchenko, Oscillation results for general second-order differential equations with damping term, J. Math. Anal. Appl. 279 (2003), 139-152.

[14] S.P. Rogovchenko and Yu.V. Rogovchenko, Oscillation of differential equations with damping, Dynam. Contin. Discrete Impuls. Systems Ser. A Math. Anal., 10 (2003), 447-461.

[15] Yu.V. Rogovchenko, Oscillation theorems for second-order differential equations with damping, Nonlinear Anal. 41 (2000), 1005-1028.

[16] Y.G. Sun, New Kamenev-type oscillation criteria for second-order nonlinear differential equations with damping, J. Math. Anal. Appl. 291 (2004), 341-351.

[17] Y.G. Sun, Note on Nasr's and Wong's papers, J. Math. Anal. Appl. 286 (2003), 363-367.

[18] A. Tiryaki and A. Zafer, Interval oscillation of a general class of second-order nonlinear differential equation with nonlinear damping, Nonlinear Anal. 60 (2005), 49-63.

[19] J.S.W. Wong, On Kamenev-type oscillation theorems for second-order differential equations with damping, J. Math. Anal. Appl. 258 (2001), 244-257.

[20] J. Yan, Oscillation theorems for second order linear differential equations with damping, Proc. Amer. Math. Soc. 98 (1986), 276-282.

[21] X. Yang, Oscillation criteria for nonlinear differential equations with damping, Appl. Math. Comput. 136 (2003), 549-557.

[22] Z. Zheng, Note on Wong's paper, J. Math. Anal. Appl. 274 (2002), 466-473. 